\title{
Vibrant times for mechanical metamaterials
}

\author{
Johan Christensen, DTU Fotonik, Department of Photonics Engineering, Technical University of Denmark, DK-2800 Kongens Lyngby, Denmark \\ Muamer Kadic and Martin Wegener, Institute of Applied Physics, Karlsruhe Institute of Technology (KIT), D-76128 Karlsruhe, Germany \\ Oliver Kraft, Institute for Applied Materials, Karlsruhe Institute of Technology (KIT), D-76128 Karlsruhe, Germany \\ Martin Wegener, Institute of Nanotechnology, Karlsruhe Institute of Technology (KIT), D-76021 Karlsruhe, Germany \\ Address all correspondence to Johan Christensen at jochri@fotonik.dtu.dk
}

(Received 15 May 2015; accepted 22 June 2015)

\begin{abstract}
Metamaterials are man-made designer matter that obtains its unusual effective properties by structure rather than chemistry. Building upon the success of electromagnetic and acoustic metamaterials, researchers working on mechanical metamaterials strive at obtaining extraordinary or extreme elasticity tensors and mass-density tensors to thereby mold static stress fields or the flow of longitudinal/transverse elastic vibrations in unprecedented ways. In this prospective paper, we focus on recent advances and remaining challenges in this emerging field. Examples are ultralight-weight, negative mass density, negative modulus, pentamode, anisotropic mass density, Origami, nonlinear, bistable, and reprogrammable mechanical metamaterials.
\end{abstract}

\section{Introduction}

Stone Age, Copper Age, Bronze Age, Iron Age: We name the eras of mankind after mechanical materials. Is the Metamaterial Age next? Physicists, material scientists, and engineers alike are already working at going beyond ("meta") what nature has given to us. Significant recent advances in threedimensional printing on the micro- and macro-scale help them to succeed. The goal is to rationally design and realize tailored artificial media with mechanical characteristics distinct from their constituents to achieve, e.g., effectively stronger, tougher, or lighter materials. In some cases, one can even go beyond what seemed to be fundamental restrictions.

As usual, the mechanics of solid bodies deals with deformations and motions resulting from external forces via Newton's law. For sufficiently small deviations from equilibrium, Hooke's law can be applied. For the paradigm of an elastic Hooke's spring, it simply states that force and extension are proportional. When considering general mechanical materials under arbitrary compression, stretching, and shearing, Hooke's law translates into rank-two tensors for stress and strain, which are connected via the rank-four elasticity tensor (Box 1). Mechanical waves in an isotropic material are an illustrative example. Generally, they can have one longitudinal polarization (like for acoustic sound waves in a gas or fluid) and two orthogonal transverse polarizations (like usually for electromagnetic waves). These three polarizations make elastodynamics even richer than acoustics and electromagnetism.

It is hard to say when the first mechanical metamaterial was conceived. However, more than a century ago, Lamb already discussed the possibility of backward waves in vibrating elastic plates. ${ }^{[1]}$ For a backward wave, mechanical energy and phase fronts move in opposite directions. Today, researchers assign negative refractive indices to such waves and speculate about the possibility of perfect lenses. Materials that expand laterally upon stretching have a negative Poisson's ratio $v$ and belong to an early class of unusual artificial mechanical materials discovered by Lakes in $1987 .{ }^{[2]}$ These structures are coined auxetics and can, e.g., be applied in shock absorbers. Auxetics have been reviewed extensively elsewhere ${ }^{[3]}$ and shall not be in the focus of the present prospective paper. Furthermore, we exclude acoustic metamaterials based on sound propagation in air or liquids, for which solids are often considered as hard-wall boundaries only (see reviews ${ }^{[4,5]}$ ). As outlined in Fig. 1, we do, however, dive into the amazing conceptual and experimental progress regarding light-weight, negative mass density, negative modulus, pentamode, anisotropic mass density, Origami, nonlinear, bistable, and reprogrammable mechanical metamaterials made in recent years.

\section{Light-weight metamaterials}

Essentially all ordinary bulk solids have a mass density above $\rho \approx 1000 \mathrm{~kg} / \mathrm{m}^{3}$. In order to expand the property space of materials toward very low densities, it is necessary to introduce voids to the material, e.g., as illustrated in Fig. 1(b). Highly regular cellular materials can be designed by the tessellation of a large number of unit cells comprising slender beams, leading to micro-architectured lattice materials, as reviewed in Fleck, Deshpande, and Ashby. ${ }^{[6]}$ Taking this approach to extreme, 
Box 1. Linear elasticity of mechanical materials.

\section{Mechanical wave propagation}

It is amazing how complex the combination of Newton's and Hooke's laws can become when applied to three-dimensional elastic solids. ${ }^{[81-83]}$ In its simplest form, Hooke's law states that the force of a spring is proportional to its extension. In more general, the force turns into the rank-two stress tensor $\stackrel{\leftrightarrow}{\sigma}$ and the extension into the rank-two strain tensor $\stackrel{\leftrightarrow}{\epsilon}$. The strain is related to the displacement vector $\vec{u}=\vec{u}(\vec{r})$ at position $\vec{r}$ via

$$
\epsilon_{l m}=\frac{1}{2}\left(\frac{\partial u_{l}}{\partial r_{m}}+\frac{\partial u_{m}}{\partial r_{l}}\right)
$$

Stress and strain are connected via the rank-four elasticity tensor $\stackrel{\leftrightarrow}{C}$, i.e.,

$$
\sigma_{i j}=\sum_{l, m=1}^{3} C_{i j l m} \epsilon_{l m} .
$$

We will review some selected properties of the elasticity tensor below. At finite frequencies, internal resonances may occur, leading to a phase-shifted response of inner masses inside of metamaterial unit cells, which can be expressed by a frequencydependent mass density $\rho(\omega)$. Different resonance frequencies may occur for different oscillation directions, in which case we get a dynamic mass-density tensor $\stackrel{\leftrightarrow}{\rho}(\omega)$. Assuming that $\stackrel{\leftrightarrow}{\rho}$ is diagonal (with generally different diagonal elements though), switching to the frequency domain, and exploiting the so-called minor symmetries $C_{i j l m}=C_{i j m l}$, Newton's law becomes

$$
-\rho_{i i} \omega^{2} u_{i}=\sum_{j, l, m=1}^{3} C_{i j l m} \frac{\partial^{2} u_{l}}{\partial r_{j} \partial r_{m}} .
$$

Elastostatics corresponds to $\omega=0$. In elastodynamics, inserting a plane-wave ansatz $\vec{u}=\vec{u}^{0} \exp (\mathrm{i} \vec{k} \cdot \vec{r})$ with wave vector $\vec{k}=\widehat{\vec{k}}|\vec{k}|=\widehat{\vec{k}} k$ leads to the Christoffel equation

$$
\left(-\delta_{i l} \rho_{i i}\left(\frac{\omega}{k}\right)^{2}+\sum_{j, m=1}^{3} C_{i j l m} \hat{k}_{j} \hat{k}_{m}\right) u_{l}^{0}=0,
$$

with Kronecker symbol $\delta_{i l}$ and phase velocity $\omega / k$. The dispersion relation $\vec{k}(\omega)$ and the three eigenpolarizations $\vec{u}^{0}$ for each direction $\widehat{\vec{k}}$ follow from the Christoffel equation. To describe metamaterials as effective media, the wavelength $2 \pi / k$ must be much larger than the metamaterial lattice constant (trivially fulfilled in the static case). In general, $\vec{k}$ and $\vec{u}^{0}$ can include angles different from $0^{\circ}$ or $90^{\circ}$. For ordinary materials, where all $C_{i j k l}>0$ and $\rho_{i i}>0,(\omega / k)^{2}$ is positive, hence the wave vector is real, which means that all mechanical waves actually propagate. In contrast, purely imaginary wave vectors corresponding to exponentially decaying contributions result if all $C_{i j k l}(\omega)>0$ and $\rho_{i i}(\omega)<0$ or if all $C_{i j k l}(\omega)<0$ and $\rho_{i i}(\omega)>0$. If components of the elasticity tensor and of the mass-density tensor are negative simultaneously, various types of unusual backward waves can result—as discussed in this prospective article.

\section{Selected properties of the elasticity tensor}

For the mathematical ideal of an isotropic material, the elasticity tensor can be represented by two independent scalar quantities, the choice of which, however, is not unique. The table gives an overview.

\begin{tabular}{lllllc}
\hline$\lambda+2 G$ & $\lambda$ & $G$ or $\mu$ & $B$ or $K$ & $v$ & $E$ \\
\hline $\begin{array}{l}\text { Longitudinal } \\
\text { modulus }\end{array}$ & $\begin{array}{c}\text { First Lamé } \\
\text { coefficient }\end{array}$ & $\begin{array}{c}\text { Second Lamé } \\
\text { coefficient/shear modulus }\end{array}$ & Bulk modulus & $\begin{array}{c}\text { Poisson's } \\
\text { ratio }\end{array}$ & Young's modulus \\
\hline$C_{11}$ & $C_{12}$ & $C_{44}=\frac{C_{11}-C_{12}}{2}$ & $\frac{C_{11}+2 C_{12}}{3}$ & $\frac{C_{12}}{C_{11}+C_{12}}$ & $C_{11}-\frac{2 C_{12}^{2}}{C_{11}+C_{12}}$ \\
\hline
\end{tabular}

Here we have used the so-called Voigt notation $C_{i j}=C_{i j i j}$ for $i, j=1,2,3$ and $C_{44}=C_{2323}$. 
The relations given in the table remain valid for cubic symmetry, but here three independent scalar quantities are generally needed to represent the elasticity tensor.

Pentamode metamaterials can be cubic, but they are special in that a single scalar quantity, the bulk modulus $B$ (= inverse of the compressibility), suffices to represent the elasticity tensor. In Voigt notation we get

$$
C_{i j}=B \forall i, j=1,2,3 ; \quad C_{i j}=0, \text { else. }
$$

Pentamode metamaterials are sometimes referred to as "meta-liquids". One should be aware though that the displacement vector, and hence the elasticity tensor, has no meaning for ordinary liquids or gases (as one does not usually track individual particles there) - in sharp contrast to pentamode metamaterials.

\section{Dynamic mass density}

For mass-and-spring resonances with eigenfrequencies $\Omega_{i}$ inside of the mechanical metamaterial unit cells, the effective behavior can be described by a mass-density tensor with components

$$
\rho_{i i}(\omega)=\rho+\Delta \rho_{i} \frac{\omega^{2}}{\Omega_{i}^{2}-\omega^{2}},
$$

where $\rho=\rho_{i i}(0) \forall i$ is the good old static scalar mass density as measured by a mass balance and $\Delta \rho_{i}$ is a quantity characterizing the oscillator strengths of the resonances. For simplicity, we have omitted damping.

metallic structures based on hollow beams with mass densities as low as $\rho=0.9 \mathrm{~kg} / \mathrm{m}^{3}$ have been realized ${ }^{[7]}$ (not counting the air mass in the voids). For comparison, the mass density of air under ambient conditions is $\rho=1.2 \mathrm{~kg} / \mathrm{m}^{3}$. The mechanical properties (such as stiffness and strength, i.e., the maximum stress before failure) of such lattice materials are obviously governed by the volume fraction of the constituent material. Moreover, details of the design, in particular the nodal connectivity (or coordination number) of the truss structure have a pronounced influence, too. Deshpande and co-workers demonstrated that, for static loading conditions, two limiting cases of micro-architectured materials can be distinguished, namely bending- and stretching-dominated structures. ${ }^{[8]}$

Examples for the former are random foams or hexagonal structures. Stretching-dominated structures typically consist of triangulated and octet-truss lattice structures in two and three dimensions, respectively. ${ }^{[9]}$ It has been shown that the normalized effective stiffness of the metamaterial $E_{\text {eff }} / E_{0}$, with $E_{0}$ being the Young's modulus of the parent or constituent material from which the trusses are made, scales according to a power law $E_{\text {eff }} / E_{0}=A\left(\sigma_{\text {eff }} / \rho_{0}\right)^{b}$ versus the relative density, which is defined as the ratio of the density of the lattice material, $\rho_{\text {eff }}$, to the one of the bulk material $\rho_{0}$. The constant $A$ is dimensionless and depends on the geometry of the lattice material and cannot exceed the value of 1 . Similarly, the relative strength scales according to $\sigma_{\text {eff }} / \sigma_{0} \propto\left(\rho_{\text {eff }} / \rho_{0}\right)^{c}$. For bendingdominated lattice materials, one finds $b=3$ and $c=2$, respectively. These exponents lead to a very unfavorable scaling of the mechanical properties with decreasing density. In contrast, one finds more favorable exponents of $b=c=1$ for stretchingdominated materials. ${ }^{[6]}$ It has been demonstrated that octet-truss metamaterials made by projection micro-stereolithography followed by nanoscale coating techniques ${ }^{[10]}$ yield an exponent near $b=1$, indeed. The fabricated structures have typical truss diameters of about $50 \mu \mathrm{m}$ and are either made of solid polymer or alumina, hollow metal, or ceramic tubes. Nevertheless, it is obvious from the equation given above that even for materials with $b=1$, the stiffness to density ratio of the metamaterial,

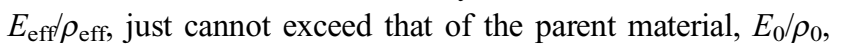
at least as long as $E_{0}$ can be considered as constant, i.e., size-independent. Therefore, the term "ultra-stiff", ${ }^{[10]}$ coined for such lattice materials should be taken with a grain of salt.

The Young's modulus of a given material can hardly be altered by changing the size of the components (or the microstructure of the material). In sharp contrast, the effective metamaterial strength can be varied by orders of magnitude. Thus, it is feasible to create ultralight materials with a higher strength-to-stiffness ratio compared with the parent material(s). For metals, such size effects originate from the suppression of dislocation motion or nucleation with decreasing dimensions, as reviewed in. ${ }^{[1]}$ For brittle materials, the strength is limited by crack nucleation at the largest flaw in the material. On macroscopic length scales, this process can be well described by Weibull statistics. In the nanometer regime, it has been argued that the strength may become insensitive to flaws. It may even reach the limit of theoretical strength, ${ }^{[12]}$ as the stress required to nucleate the crack from a flaw increases with decreasing dimensions. This reasoning assumes that the size of the largest flaw does not exceed the smallest dimension of a component, e.g., the thickness of a thin film or a small platelet. Based on this approach, it has been demonstrated that the miniaturization of lattice materials with trusses in the submicron regime creates 


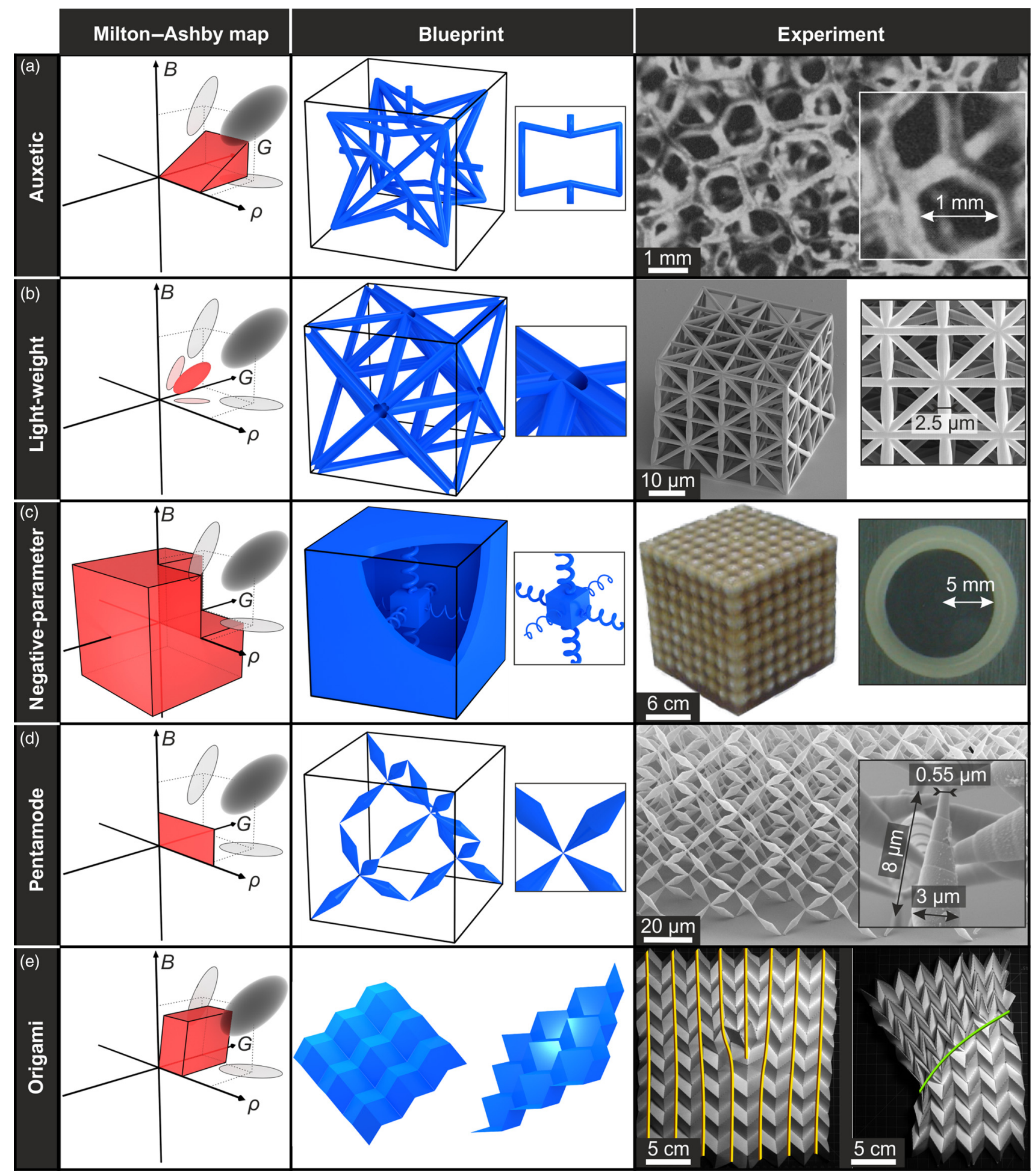

Figure 1. Overview on mechanical metamaterials. The five rows illustrate (a) auxetic, (b) light-weight, (c) negative-parameter (i.e., negative mass density and/or moduli at finite frequency $\omega \neq 0$ ), (d) pentamode, and (e) Origami mechanical metamaterials. The left column shows a combination of the Milton map (bulk modulus $B$ versus shear modulus $G$ ) and the Ashby map (one elastic modulus versus mass density $\rho$ ). The parameters are zero at the crossing of the three arrows, pointing into the positive directions. In each entry, ordinary solids (black) are compared with the corresponding metamaterials (red). The center column exhibits blueprints of (extended) unit cells highlighting characteristic structural elements, the right column optical or electron micrographs of fabricated structures. These metamaterials can (a) be easily compressible, yet not easily deformable; (b) be light-weight, yet ultrastrong; (c) exhibit complete band gaps or support backward waves; (d) be easily deformable, but not easily compressible; (e) be deployable, light-weight, bistable, and reprogrammable. Applications could be as (a) shock absorbers, (b) support structures, (c) reflectors or concentrators, (d) mechanical cloaks, and (e) structures for space missions. Figures reproduced with permission: (a) (c) 1987 AAAS; (b) @ 2014 PNAS; (c) @ 2000 AAAS; (d) @ 2012 AIP; (e) @ 2014 AAAS. 
materials with strength-to-density ratios $\sigma_{\text {eff }} \rho_{\text {eff }}$ exceeding those of ordinary as well as that of engineered cellular materials. ${ }^{[13-15]}$ The fabrication of these metamaterials has become possible by three-dimensional dip-in direct laser writing of polymer templates. $^{[16]}$ Subsequently, these templates are coated via atomic-layer deposition of alumina, with layer thicknesses in the range of $10-100 \mathrm{~nm}$.

$\mathrm{In}^{[13]}$ the polymer has been removed after fabrication, and the final lattice structure hence consists of hollow alumina tubes. It was demonstrated that the scaling exponent, $c$, for the strength for such hollow-tube octet-truss structures is smaller than 2, indicating that it is possible to make use of the stretching-dominated geometry although the trusses are hollow. For such structures, three competing failure mechanisms can be distinguished, including fracture of the tube wall, beam buckling of a truss member, and shell buckling of the tube wall. ${ }^{[17]}$ For alumina wall thicknesses $<50 \mathrm{~nm}$, the structures showed a significant recoverability from compressive strains as large as $50 \%$. On this basis, it has been argued that local buckling becomes the dominating factor for very thin alumina shells. ${ }^{[17]}$

Bauer et al. ${ }^{[14]}$ took a somewhat different approach by fabricating lattice materials in which the polymer remains as core inside the trusses. Here, the strength of the structure is limited by local buckling of trusses, which occurs when a stiff alumina shell fails by cracking. The strength of these core-shell polymer-ceramic composites is estimated to be of the order of GPa. ${ }^{[15]}$ The effective strength of these metamaterials reaches up to several $100 \mathrm{MPa}$.

These examples just touch upon the potential strengths of lattice materials. Nevertheless, they do demonstrate convincingly that the outlined ${ }^{[6]}$ expansion in mechanical property space by design of lattice materials is achievable in reality, especially if one takes advantage of size effects. With foreseeable further improvements regarding three-dimensional micro- and nano-manufacturing, hierarchical micro-architectured lightweight materials with tailored stiffness, strength, and toughness with the "overall macroscopic volumes" required for real-world applications are expected to become available in the near future. So far, these small overall structures should rather be considered as prototypes.

\section{Negative mass densities and negative elastic moduli}

Under the static conditions discussed so far, the mass density of a material cannot be negative. Likewise, the elastic moduli must be positive for any stable and unconstrained material. Negative effective parameters are allowed though at finite frequencies near local resonances. These can have small resonance frequencies equivalent to wavelengths much larger than the lattice constant of a periodic metamaterial structure. Under these conditions, a discussion in terms of effective mechanical material parameters is possible. A negative mass density $\rho$ means that an elastic body accelerates out of phase with respect to a harmonically varying driving force. For a negative bulk modulus
$B$, the body would compress upon dynamic stretching. Combining $B<0$ and $\rho<0$ leads to the counterpart of doublenegative or negative-index metamaterials in optics ${ }^{[5,18]}$ (also see Box 1).

For example, spherical metal cores coated with a compliant rubber shell, packed to a simple-cubic lattice in a host material have been considered early on as depicted in Fig. 1(c). ${ }^{[19]}$ Each core-shell unit forms a simple mass- and spring model, which can exhibit a Mie-type resonance frequency far below the Bragg resonance frequency of the lattice. ${ }^{[19,20]}$ Depending of the order of these resonances, negative effective values of the mass density and/or of the elastic moduli can be accomplished. More specifically, numerical predictions have shown that cylindrical or spherical cores embedded into a host material can yield collective Mie oscillations that give rise to negative longitudinal moduli $\lambda+2 G$, negative shear moduli $G$, or negative bulk moduli $B .{ }^{[21]}$ A large material contrast between host material and inclusion helps to obtain pronounced resonances. An additional coating layer can be used to broaden the spectral response-if desired. By combining these designed ingredients, negative refraction and anomalous dispersion can be achieved for specific polarizations. Combined with a negative mass density, ${ }^{[22,23]}$ the mechanical energy-flow (Poynting) vector points into a direction opposite to that of the phase-velocity vector. Corresponding experiments have used rubber spheres suspended in water ${ }^{[24]}$ (taking advantage of the high-velocity contrast permitting to omit shear contributions) or silicone rubber embedded in a water-based gel host, leading to a negative index of refraction. ${ }^{[25]}$

Other publications predict a negative bulk modulus transition induced by interplay of different force potentials. ${ }^{[26]}$

Furthermore, a chiral route toward negative elastic refraction has lately been discussed. ${ }^{[27-31]}$ As usual, an object is called chiral if it cannot be brought into congruence with its mirror image by translations and rotations. Helices or screws are paradigms. Chiral or handed media can exhibit activity (like in optics), which means that an incident linear polarization is rotated upon propagation through the medium. Earlier studies suggested the construction of acoustically active media by embedding chiral microstructures in a host material, ${ }^{[27-29]}$ which have been realized in cellular lattices that exhibit auxetic properties as well. ${ }^{[30]}$ Other authors have perforated chiral microstructures into thin steel plates and were able to experimentally detect negative refraction of a longitudinally polarized wave. ${ }^{[31]} \mathrm{By}$ combining dipolar-like and rotational resonances of the oscillating chiral center piece, a collective mode is induced that exhibits both negative effective mass density and negative longitudinal modulus.

\section{Pentamode metamaterials}

Transformation optics is a powerful and intuitive design principle, ${ }^{[32,33]}$ which maps fictitious coordinate transformations onto actual material-parameter distributions, thereby achieving specific desired functions. Invisibility cloaking has been a paradigm, because this function appeared impossible for a long 
time. Unfortunately, the three-dimensional linear elastomechanical equations (see Box 1), unlike the Maxwell equations in electromagnetism, are not form-invariant under general coordinate transformations, at least not for the elasticity tensors of ordinary solids and for scalar mass densities. ${ }^{[32]}$ Thus, transformation optics cannot easily be translated to mechanics - neither to the static nor to the dynamic case. Flexural waves in thin plates $^{[34]}$ or seismic Rayleigh waves on the earth's surface ${ }^{[35]}$ are notable exceptions. Both examples are effectively twodimensional and exhibit a single approximately transverse polarization. The direct lattice-transformation approach ${ }^{[36]}$ also uses coordinate transformations for design, however, without making reference to effective mechanical material parameters. So far, this approximate approach has been limited to twodimensional static situations though. ${ }^{[36]}$

In three dimensions, a solution to the lack of form-invariance in mechanics is based on pentamode metamaterials. ${ }^{[37]}$ Their two-dimensional cousins are bimode metamaterials. ${ }^{[38]}$ Pentamode materials can be represented by a pseudo elasticity tensor with only one non-zero eigenvalue that is of the pure compression type. Loosely speaking, pentamode metamaterials can be seen as meta-liquids or anti-auxetics with $G=0$ yet $B \neq 0$ (also see Box 1). It is interesting to note in passing that all conceivable linear elasticity tensors of three-dimensional materials can, in principle, be synthesized on the basis of pentamode metamaterials. ${ }^{[39]}$ Specific three-dimensional microstructures were independently suggested many years ago by Sigmund ${ }^{[40]}$ using numerical topology optimization and by Milton and Cherkaev. ${ }^{[39]}$ Their considerations are analytical and include isotropic as well as anisotropic versions. For the isotropic case, they suggested a lattice of double-cone elements, touching only at singular points. These points form a diamond lattice as illustrated in Fig. 1(d). However, fabrication of corresponding threedimensional polymer microstructures ${ }^{[41]}$ has become possible only recently by virtue of three-dimensional dip-in direct laser writing. ${ }^{[16]}$ Herein, the touching points are approximated by small but finite connections. If their diameter is of the order of $1 \%$ of the metamaterial lattice constant, the effective metamaterial bulk modulus $B$ can be more than three orders of magnitude larger than the effective shear modulus $G .{ }^{[41]}$ Hence, Poisson's ratio comes very close to $v=0.5$ from below. This behavior has been confirmed by static experiments on macroscopic structures ${ }^{[42]}$ and by numerical calculations of the phonon band structure, ${ }^{[4]}$ in which the transversely polarized shear modes appear as flat branches. In a large-frequency region, even only a single isotropic longitudinal phonon or vibration mode can be achieved. ${ }^{[43]}$

We note in passing that pentamode behavior with $B / G \gg 1$ has also been predicted theoretically for disordered jammed packings of harmonic particles. ${ }^{[4]}$

Owing to stress concentration close to the double-cone tips, the pentamode metamaterial bulk modulus is mainly determined by the tip diameter. In contrast, the thick part of the double-cones is of much lesser importance. ${ }^{[45]}$ Thus, presently experimentally accessible pentamode metamaterials have volume filling fractions as low as $\approx 1 \%$, i.e., they can be extremely light-weight (see above). In fact, the mass density can be adjusted largely independently via the diameter of the thick part of the double-cones over a fairly large range. Equivalently, this allows for independently tailoring the phonon phase velocity and wave impedance. ${ }^{[45]}$ Ultra-compliant heavy metamaterials, the opposite of strong ultralight metamaterials discussed above, are an example.

\section{Anisotropic versions of pentamode metamaterials}

Anisotropic versions of pentamode ${ }^{[46]}$ (and bimode ${ }^{[47]}$ ) mechanical metamaterials as well as effectively anisotropic laminates composed of isotropic pentamode metamaterials have been considered as well. ${ }^{[4]}$ As in any laminate metamaterial, ${ }^{[48]}$ the anisotropy originates from the layering. If the bulk moduli of the layers are identical, whereas their mass densities are different, an anisotropic dynamic mass-density tensor $\stackrel{\leftrightarrow}{\rho}$ arises. ${ }^{[45]}$ Likewise, an anisotropic elasticity tensor $\stackrel{\leftrightarrow}{C}$ results for the opposite configuration. This setup has been used for early experimental demonstrations of static elastic ("unfeelability") cloaking in cylindrical core-shell structures. ${ }^{[49]}$ Herein, the shell around a massive stiff hollow core has a particular smaller bulk modulus than the surrounding, whereas the mass densities of shell and surrounding are nearly identical.

Effective anisotropy can also be achieved by making the unit cell itself less symmetrical than the three-dimensional pentamode diamond lattice ${ }^{[46]}$ (or the two-dimensional hexagonal lattice, ${ }^{[4]}$ respectively). For example, one double-cone connection point can be shifted along the space diagonal of the facecentered cubic unit cell. ${ }^{[39,46]}$ However, one should no longer call these structures pentamode (or bimode) because they have less than five (two) easy modes - even for small doublecone connection diameters. Correspondingly, anisotropy is inherently connected to finite shear forces. Maximum anisotropy is even connected to zero Poisson's ratio and a transition toward auxetic behavior. ${ }^{[46]}$

\section{Anisotropic mechanical metamaterials}

Crystalline materials rarely exhibit truly isotropic mechanical properties. Even a simple-cubic lattice in the long-wavelength limit generally leads to anisotropic mechanical behavior (also see Box 1). Ideal pentamode metamaterials discussed above are one notable exception, isotropic auxetics with $v=-1$ (also coined dilational metamaterials) another one. ${ }^{[50]}$ In general, specific anisotropies may be desirable as just discussed for transformation elastodynamics.

Broadly speaking, anisotropic mechanical metamaterials can be realized by laminates, ${ }^{[48]}$ fiber composites, and perforations, to name just a few established concepts. In anisotropic metamaterials, the displacement vectors are not always purely parallel or perpendicular with respect to the propagation wave vector. Thus, the purely longitudinal and the two transverse polarizations of isotropic materials turn into one quasilongitudinal and two quasi-transverse modes. Anisotropy can 
be expressed through the stiffness tensor (see Box 1). At finite frequencies, it can also be mapped onto the mass-density tensor $\stackrel{\leftrightarrow}{\rho}$, which leads to a modified version of Newton's Second Law. ${ }^{[51]}$

Milton and Willis ${ }^{[51]}$ suggested that an anisotropic massdensity tensor can be created by introducing anisotropy to local resonances in the unit cell (or motif) rather than by the metamaterials translational lattice. A simple example is an oscillating internal mass attached to different Hooke's springs in the three spatial directions. ${ }^{[52]}$ Lai and co-workers modified this approach by introducing multiple masses into the unit cell. This crystal with simple-cubic translational lattice exhibits resonances with negative values of the scalar mass density and three independent negative elastic moduli. Depending on the propagation direction of the wave, this structure can mimic a fluid permitting only longitudinal waves or a hybrid fluid-solid behavior with longitudinal and shear wave propagation. ${ }^{[53]}$

Iso-frequency surfaces are an alternative way for illustrating the fingerprint of anisotropic mechanical metamaterial properties. The surface shape depends on the magnitude and the sign of the individual elastic parameters. For mixed signs, hyperbolic dispersion relations result that lead to negative refraction, which has recently been shown experimentally in airborne acoustic metamaterials. ${ }^{[54]}$ A prominent application of mechanical metamaterials with hyperbolic dispersion is the so-called hyperlens. Evanescent waves emitted by a subwavelength source are radiated by conversion into propagating elastic waves in the curved hyperlens. Recent experiments ${ }^{[5]}$ show that these partial waves can even be magnified and guided toward the far field.

Following the original idea of Milton and Willis, several experimental efforts have worked towards constructing metamaterials with an effectively anisotropic mass-density tensor. Theoretical efforts were devoted to study the elastic response of locally resonant structures consisting of asymmetrically coated elliptical cores embedded in a rigid background. As expected, the in-plane mechanical displacements along the main axes were different. Through modified Newton's law, this behavior can be mapped onto an effectively anisotropic massdensity tensor. ${ }^{[56]}$ The same conclusions were reached a few years later for a similar structure through analytical predictions and finite-element calculations. ${ }^{[57]}$ Importantly, the massdensity tensor always merges into the good old scalar mass density in the low-frequency limit. Intuitively, at mechanical standstill, the mass of a body has no orientation. These ideas, which were tested in experiments comprising resonators with elliptical micro-perforated coatings, showed good agreement between simulated and measured effective mass densities. Furthermore, transmission measurements for wave propagation along two principal directions demonstrated the existence of band gaps induced by the respective singly negative components of the mass-density tensor. ${ }^{[58]}$

By clamping the mass of the coated core in the aforementioned metamaterial, the mechanical wave behaves just like light in a free-electron Drude metal. In other words, the resonance frequency goes to zero. Equivalently, the effective mass density at low frequencies goes to $-\infty$ and remains negative below a cut-off frequency. This behavior is analogous to that of a membrane in a narrow channel. ${ }^{[59]}$ Instead of a channel, a soft solid is filled into the holes of a perforated rigid and immovable material to constitute a mechanical metamaterial with extreme anisotropy. ${ }^{[60]}$ The effective mass-density-tensor component along the perforations is of the above Drude form, whereas the in-plane component goes to $+\infty$ corresponding to an immovable body. These systems have shown their potential for spoof acousto-elastic surface waves and transmission enhancement in thin plates. ${ }^{[61,62]}$ The complementary medium composed of rigid bars in a soft background works equally well. ${ }^{[63]}$ In a similar configuration of square rods in a non-rigid host, one also obtains a complex anisotropy for both, the mass density and the elasticity tensor. ${ }^{[64]}$

\section{Origami and programmable metamaterials}

Origami is the traditional Asian art of paper folding, Kirigami additionally allows for introducing cuts into the paper. Today, by using free available computer programs such as "TreeMaker", ${ }^{[65]}$ one can design the Origami folding patterns of almost arbitrary complex three-dimensional structures - including Origami (Kirigami) mechanical metamaterials. ${ }^{[66,67]}$ Instead of paper, one can likewise start from a thin polymer sheet with indentations defining the creases or from rigid structures with hinges instead of the creases. ${ }^{[6]}$ Effectively, one can think of the creases as torsional Hooke's springs. ${ }^{[69]}$ Temperature-responsive polymergels instead of paper are yet another option. ${ }^{[70]}$

Paper is an inextensible constituent material, yet the metamaterials made thereof can effectively be highly flexible. For the simple example of a one-dimensional accordion folding, it is immediately clear that the Origami metamaterial can be compressed by nearly $100 \%$. The effective elastic moduli, mass density, and wave velocity clearly depend on the degree of compression. In this fashion, the metamaterial properties are tunable. One may even be able to open or close phonon stop bands or band gaps. Additionally, Origami metamaterials are deployable, which is an attractive feature for space applications. Indeed, the famous Miura folding has originally been engineered to deploy satellite solar panels in space. ${ }^{[71]}$ Miura metamaterials can have negative or positive effective Poisson's ratios. ${ }^{[66,72-74]}$ In the assembled state, Origami metamaterials can be extremely light-weight, yet rigid. In principle, most lithographically fabricated metamaterials discussed above could be made by Origami as well.

The nonlinear mechanical properties of Origami metamaterials are even more interesting: One finds bistable behavior of each unit cell. ${ }^{[70,73]}$ Switching one cell leads to a defect in the lattice. ${ }^{[73]}$ Importantly, the effective linear metamaterial mechanical properties around such a stable state can be significantly different for the different stable states. This behavior allows for going beyond tuning ${ }^{[75-77]}$ in that one can rationally 
and reversibly choose a stable state. The metamaterial will then stay in that state without further external stimulus (in contrast, pure tuning requires maintaining the stimulus); see Fig. 1(e). In this sense, the metamaterial properties are programmable or reprogrammable $^{[73]}$ (also se ${ }^{[68,78,79]}$ ). If the constituent material had an infinitely large stretching modulus, the Miura folding would exhibit only one degree of freedom ${ }^{[66,71]}$; bistability could not occur. However, real materials can bend and the total energy is the sum of crease and bending energy, allowing for bistable behavior. ${ }^{[70,73]}$ A simple example of a building block that exhibits bistable behavior is a circular piece of paper folded once along its diameter. Buckling up or down leads to two stable states.

\section{Conclusions and perspectives}

Mechanical metamaterials can be seen as "matter made to order". Scientists turn into artists or designers when conceiving such artificial solids. The field is simply fun and cool. The resulting properties addressed in this prospective article range from highly strange via extreme to mind-boggling. But will this field really have an impact on our every-day life in the not-too-distant future? A "yes" requires progress with respect to at least two frontiers.

First, fabrication needs another boost. Three-dimensional printing techniques can now make thousands of complex shaped microscopic metamaterial unit cells in reasonable time, but real-world applications likely demand yet more than a thousand times more. Currently, three-dimensional laserbased additive manufacturing attracts huge attention worldwide. Once this technology makes the anticipated jump from prototyping to industrial mass fabrication, mechanical metamaterials could be a huge beneficiary.

Second, "killer" properties and functions may still need to be identified. Have we thought big enough? Has the community focused too much on the linear elastic regime? We have, for example, mentioned mechanical bistability. Bistable switching in the stress-strain curve under strain control corresponds to hysteretic behavior under stress control. Hysteretic behavior means that the metamaterial absorbs energy in one cycle of compression and expansion. The energy is given by the enclosed area. Such mechanical metamaterials could thus be used as reversible shock absorbers, i.e., they would absorb the energy of an impact, yet, unlike for a car bumper, maintain their structural integrity. Ideally, this mechanical nonlinearity would originate exclusively from the metamaterial structure and not from the constituent materials. In this spirit, recent theoretical work has mathematically constructed mechanical metamaterials with any desired nonlinearity of the Poisson's ratio. ${ }^{[80]}$

In brief, bringing mechanical metamaterials from cool science towards real-world products requires that we keep on working hard and let our imagination fly.

\section{Acknowledgments}

We thank Steve Cummer (Duke University), Peter Gumbsch (KIT), Graeme W. Milton (University of Utah), Jose
Sanchez-Dehesa (Polytechnical University of Valencia), and Morten Willatzen (Technical University of Denmark) for stimulating discussions. J. C. gratefully acknowledges financial support from the Danish Council for Independent Research and a Sapere Aude grant (no. 12-134776). O. K. is grateful for support by the Robert Bosch foundation. M. W. acknowledges support by the Helmholtz Association through the Program Science and Technology of Nanosystems (STN) and the Karlsruhe School of Optics \& Photonics (KSOP). M. K. and M. W. acknowledge support by the Hector Fellow Academy.

\section{References}

1. H. Lamb: On group-velocity. Proc. Lond. Math. Soc. 1, 473 (1904).

2. R.S. Lakes: Foam structures with negative Poisson's ratio. Science $\mathbf{2 3 5}$, 1038 (1987).

3. G.N. Greaves, A.L. Greer, R.S. Lakes, and T. Rouxel: Poisson's ratio and modern materials. Nat. Mater. 10, 823 (2011).

4. M. Moldovan: Sound and heat revolutions in phononics. Nature 503, 209 (2013).

5. M. Kadic, T. Bückmann, R. Schittny, and M. Wegener: Metamaterials beyond electromagnetism. Rep. Prog. Phys. 76, 126501 (2013).

6. N.A. Fleck, V.S. Deshpande, and M.F. Ashby: Micro-architectured materials: past, present and future. Proc. R. Soc. A 466, 2495 (2010).

7. T.A. Schaedler, A.J. Jacobsen, A. Torrents, A.E. Sorensen, J. Lian, J.R. Greer, L. Valdevit, and W.B. Carter: Ultralight metallic microlattices. Science 334, 962 (2011).

8. V.S. Deshpande, M.F. Ashby, and N.A. Fleck: Foam topology: bending versus stretching dominated architectures. Acta Mater. 49, 1035 (2001).

9. V.S. Deshpande, M.F. Ashby, and N.A. Fleck: Effective properties of the octet-truss lattice material. J. Mech. Phys. Solids 49, 1747 (2001).

10.X. Zheng, H. Lee, T.H. Weisgraber, M. Shusteff, J. DeOtte, E.B. Duoss, J.D. Kuntz, M.M. Biener, Q. Ge, J.A. Jackson, S.O. Kucheyev, N. X. Fang, and C.M. Spadaccini: Ultralight, ultrastiff, mechanical metamaterials. Science 344, 1373 (2014).

11. O. Kraft, P.A. Gruber, R.M. Mönig, and D. Weygand: Plasticity in confined dimensions. Annu. Rev. Mater. Res. 40, 293 (2010).

12. H. Gao, B. Ji, I.L. Jäger, E. Arzt, and P. Fratzl: Materials become insensitive to flaws at nanoscale: lessons from nature. Proc. Nat. Acad. Sci. USA 100, 5597 (2003).

13. L.R. Meza, S. Das, and J.R. Greer: Strong, lightweight, and recoverable three-dimensional ceramic nanolattices. Science 345, 1322 (2014).

14.J. Bauer, S. Hengsbach, I. Tesari, R. Schwaiger, and 0. Kraft: High-strength cellular ceramic composites with 3D microarchitecture. Proc. Nat. Acad. Sci. USA 111, 2453 (2014).

15. J. Bauer, A. Schroer, R. Schwaiger, I. Tesari, C. Lange, L. Valdevit, and 0 . Kraft: Push-to-pull tensile testing of ultra-strong nanoscale ceramicpolymer composites made by additive manufacturing. Extreme Mech. Lett. 3, 105 (2015).

16. T. Bückmann, N. Stenger, M. Kadic, J. Kaschke, A. Frölich, T. Kennerknecht, C. Eberl, M. Thiel, and M. Wegener: Tailored 3D mechanical metamaterials made by dip-in direct-laser-writing optical lithography. Adv. Mater. 24, 2710 (2012).

17. L. Valdevit, A.J. Jacobsen, J.R. Greer, and W.B. Carter: Protocol for the optimal design of multifunctional structures: from hypersonics to microarchitected materials. J. Am. Ceram. Soc. 94, s15 (2011).

18. C.M. Soukoulis and M. Wegener: Past achievements and future challenges in the development of three-dimensional photonic metamaterials. Nat. Photonics 5, 523 (2011).

19.Z. Liu, X. Zhang, Y. Mao, Y.Y. Zhu, Z. Yang, C.T. Chan, and P. Sheng: Locally resonant sonic materials. Science $\mathbf{2 8 9}, 1734$ (2000).

20.H.H. Huang, C.T. Sun, and G.L. Huang: On the negative effective mass density in acoustic metamaterials. Int. J. Eng. Sci. 47, 610 (2009).

21. Y. Wu, Y. Lai, and Z.-Q. Zhang: Effective medium theory for elastic metamaterials in two dimensions. Phys. Rev. B 76, 205313 (2007). 
22. Y. Ding, Z. Liu, C. Qiu, and J. Shi: Metamaterials with simultaneously negative bulk modulus and mass density. Phys. Rev. Lett. 99, 093904 (2007).

23. Y. Wu, Y. Lai, and Z.-Q. Zhang: Elastic metamaterials with simultaneously negative effective shear modulus and mass density. Phys. Rev. Lett. 107, 105506 (2011).

24. J. Li and C.T. Chan: Double-negative acoustic metamaterial. Phys. Rev. E 70, 055602(R) (2004).

25. T. Brunet, A. Merlin, B. Mascaro, K. Zimny, J. Leng, 0. Poncelet, C. Aristégui, and 0 . Mondain-Monval: Soft 3D acoustic metamaterials with negative index. Nat. Mater. 14, 384 (2015).

26.Z.G. Nicolaou and A.E. Motter: Mechanical metamaterials with negative compressibility transitions. Nat. Mater. 11, 608 (2012).

27. V.V. Varadan, A. Lakhtakia, and V.K. Varadan: Geometry can be the basis for acoustic activity (a la optical activity) in composite media. J. Wave Mater. Interact. 1, 315 (1986).

28. A. Lakhtakia, V.V. Varadan, and V.K. Varadan: Elastic wave propagation in noncentrosymmetric, isotropic media: dispersion and field equations. $J$. Appl. Phys. 63, 5246 (1988).

29. S.F. Nagle, A. Lakhtakia, and W. Thompson: Modal structures for axial wave propagation in a continuously twisted structurally chiral medium. J. Acoust. Soc. Am. 97, 42 (1995).

30. K.F. Tee, A. Spadoni, F. Scarpa, and M. Ruzzene: Wave propagation in auxetic tetrachiral honeycombs. ASME J. Vibr. Acoust. 132, 031007 (2010).

31. R. Zhu, X.N. Liu, G.K. Hu, C.T. Sun, and G.L. Huang: Negative refraction of elastic waves at the deep-subwavelength scale in a single-phase metamaterial. Nat. Commun. 5, 5510 (2014).

32. G.W. Milton, M. Briane, and J.R. Willis: On cloaking for elasticity and physical equations with a transformation invariant form. New J. Phys. 8, 248 (2006).

33. J.B. Pendry, D. Schurig, and D.R. Smith: Controlling electromagnetic fields. Science 312, 1780 (2006).

34. N. Stenger, M. Wilhelm, and M. Wegener: Experiments on elastic cloaking in thin plates. Phys. Rev. Lett. 108, 014301 (2012).

35. S. Brule, E.H. Javelaud, S. Enoch, and S. Guenneau: Experiments on seismic metamaterials: molding surface waves. Phys. Rev. Lett. 112, 133901 (2014).

36. T. Bückmann, M. Kadic, R. Schittny, and M. Wegener: Mechanical cloak design by direct lattice transformation. Proc. Natl. Acad. Sci. USA 112, 4930 (2015).

37. A.N. Norris: Acoustic cloaking theory. Proc. R. Soc. A 464, 2411 (2008).

38. A.N. Norris: Acoustic metafluids. J. Acoust. Soc. Am. 125, 839 (2009).

39. G.W. Milton and A. Cherkaev: Which elasticity tensors are realizable? J. Eng. Mater. Technol. 117, 483 (1995).

40. O. Sigmund: Tailoring materials with prescribed elastic properties. Mech. Mater. 20, 351 (1995).

41. M. Kadic, T. Bückmann, N. Stenger, M. Thiel, and M. Wegener: On the practicability of pentamode mechanical metamaterials. Appl. Phys. Lett. 100, 191901 (2012).

42. A. Martin, M. Kadic, R. Schittny, T. Bückmann, and M. Wegener: Phonon band structures of three-dimensional pentamode metamaterials. Phys. Phys. B 86, 155116 (2012).

43. R. Schittny, T. Bückmann, M. Kadic, and M. Wegener: Elastic measurements on macroscopic three-dimensional pentamode metamaterials. Appl. Phys. Lett. 103, 231905 (2013).

44.W.G. Ellenbroek, Z. Zeravcic, W. van Saarloos, and M. van Hecke: Non-affine response: jammed packings vs. spring networks. Europhys. Lett. 87, 34004 (2009).

45. M. Kadic, T. Bückmann, R. Schittny, P. Gumbsch, and M. Wegener: Pentamode metamaterials with independently tailored bulk modulus and mass density. Phys. Rev. Appl. 2, 054007 (2014).

46. M. Kadic, T. Bückmann, R. Schittny, and M. Wegener: On anisotropic versions of three-dimensional pentamode metamaterials. New J. Phys. 15, 023029 (2013)

47.C.N. Layman, C.J. Naify, T.P. Martin, D.C. Calvo, and G.J. Orris: Highly-anisotropic elements for acoustic pentamode applications. Phys. Rev. Lett. 111, 024302 (2013).

48. G.W. Milton: The Theory of Composites (Cambridge University Press, Cambridge, 2002).

49. T. Bückmann, M. Thiel, M. Kadic, R. Schittny, and M. Wegener: An elastomechanical unfeelability cloak made of pentamode metamaterials. Nat. Commun. 5, 4130 (2014).
50.T. Bückmann, R. Schittny, M. Thiel, M. Kadic, G.W. Milton, and M. Wegener: On three-dimensional dilational elastic metamaterials. New J. Phys. 16, 033032 (2014).

51. G.W. Milton and J.R. Willis: On modifications of Newton's second law. Proc. R. Soc. A 463, 855 (2007).

52. T. Bückmann, M. Kadic, R. Schittny, and M. Wegener: Mechanical metamaterials with anisotropic effective mass density tensor made from one constituent. Phys. Status Solidi B, in press (2015). D0I: 10.1002/ pssb.201451698.

53. Y. Wu, Y. Lai, P. Sheng, and Z.-Q. Zhang: Hybrid elastic solids. Nat. Mater. 10, 620 (2011).

54. V.M. Garcia-Chocano, J. Christensen, and J. Sanchez-Dehesa: Negative refraction and energy funneling by hyperbolic materials: an experimental demonstration in acoustics. Phys. Rev. Lett. 112, 144301 (2014).

55. J.H. Oh, H.M. Seung, and Y.Y. Kim: A truly hyperbolic elastic metamaterial lens. Appl. Phys. Lett. 104, 073503 (2014).

56. Y. Gu, X. Luo, and H. Ma: Low frequency elastic wave propagation in two dimensional locally resonant phononic crystal with asymmetric resonator. J. Appl. Phys. 105, 044903 (2009).

57. A.P. Liu, R. Zhu, X.N. Liu, G.K. Hu, and G.L. Huang: Multi-displacement microstructure continuum modeling of anisotropic. Wave Motion 49, 411 (2012).

58. R. Zhu, X.N. Liu, G.L. Huang, H.H. Huang, and C.T. Sun: Microstructural design and experimental validation of elastic metamaterial plates with anisotropic mass density. Phys. Rev. B 86, 144307 (2012).

59.R. Fleury and A. Alu: Extraordinary sound transmission through density-near-zero ultranarrow channels. Phys. Rev. Lett. 111, 055501 (2013).

60. S. Yao, X. Zhou, and G. Hu: Investigation of the negative-mass behaviours occurring below a cut-off frequency. New J. Phys. 12, 103025 (2010).

61. R. Hao, C. Qiu, Y. Ye, C. Li, H. Jia, M. Ke, and Z. Liu: Transmission enhancement of acoustic waves through a thin hard plate embedded with elastic inclusions. Appl. Phys. Lett. 101, 021910 (2012).

62. J. Christensen, Z. Liang, and M. Willatzen: Metadevices for the confinement of sound and broadband double-negativity behavior. Phys. Rev. $B$ 88, 100301(R) (2013).

63. P. Peng, C. Qiu, Z. Liu, and Y. Wu: Controlling elastic waves with small phononic crystals containing rigid inclusions. Eur. Phys. Lett. 106, 46003 (2014)

64.J. Flores-Mendez and F. Perez-Rodriguez: Metasolid with anisotropic mass density. Eur. Phys. Lett. 103, 54001 (2013).

65. R.J. Lang: Origami Design Secrets, 2nd ed. (CRC Press, Boca Raton, FL, 2013).

66. M. Schenk and S.D. Guest: Geometry of Miura-folded metamaterials. Proc. Natl. Acad. Sci. USA 110, 3276 (2013).

67. T. Castle, Y. Cho, X. Gong, E. Jung, D.M. Sussman, S. Yang, and R.D. Kamien: Making the cut: lattice kirigami rules. Phys. Rev. Lett. 113, 245502 (2014).

68. S. Waitukaitis, R. Menaut, B.G. Chen, and M. van Hecke: Origami multistability: from single vertices to metasheets. Phys. Rev. Lett. 114, 055503 (2015).

69. F. Lechenault, B. Thiria, and M. Adda-Bedia: Mechanical response of a creased sheet. Phys. Rev. Lett. 112, 244301 (2014).

70. J.L. Silverberg, J.-H. Na, A.A. Evans, B. Liu, T.C. Hull, C.D. Santangelo, R.J. Lang, R.C. Hayward, and I. Cohen: Origami structures with a critical transition to bistability arising from hidden degrees of freedom. Nat. Mater. 14, 389 (2015).

71. K. Miura: Method of packaging and deployment of large membranes in space. Inst. Space Astronaut. Sci. Rep. 618, 1 (1985).

72. Z.Y. Wei, Z.V. Guo, L. Dudte, H.Y. Liang, and L. Mahadevan: Geometric mechanics of periodic pleated origami. Phys. Rev. Lett.110, 215501 (2013).

73.J.L. Silverberg, A.A. Evans, L. McLeod, R. Hayward, T. Hull, C.D. Santangelo, and I. Cohen: Using origami design principles to fold reprogrammable mechanical metamaterials. Science 345, 647 (2014).

74. C. Lv, D. Krishnaraju, G. Konjevod, H. Yu, and H. Jiang: Origami based mechanical metamaterials. Sci. Rep. 4, 5979 (2014).

75. P. Wang, F. Casadei, S. Shan, J.C. Weaver, and K. Bertoldi: Harnessing buckling to design tunable locally resonant acoustic metamaterials. Phys. Rev. Lett. 113, 014301 (2014). 
76. S. Shan, S.H. Kang, P. Wang, C. Qu, S. Shian, E.R. Chen, and K. Bertoldi: Harnessing multiple folding mechanisms in soft periodic structures for tunable control of elastic waves. Adv. Funct. Mater. 24, 4935 (2014).

77. M. Willatzen and J. Christensen: Acoustic gain in piezoelectric semiconductors at $\varepsilon$-near-zero response. Phys. Rev. B 89, 041201(R) (2014).

78. B. Florijn, C. Coulais, and M. van Hecke: Programmable mechanical metamaterials. Phys. Rev. Lett. 113, 175503 (2014).

79. S.H. Kang, S. Shan, A. Košmrlj, W.L. Noorduin, S. Shian, J.C. Weaver, D. R. Clarke, and K. Bertoldi: Complex ordered patterns in mechanical instability induced geometrically frustrated triangular cellular structures. Phys. Rev. Lett. 112, 098701 (2014).

80. G.W. Milton: Complete characterization of the macroscopic deformations of periodic unimode metamaterials of rigid bars and pivots. J. Mech. Phys. Sol. 61, 1543 (2013).

81.0. Rand and V. Rovenski: Analytical Methods in Anisotropic Elasticity (Birkhäuser, Basel, 2005).

82. B. Banerjee: An Introduction to Metamaterials and Waves in Composites (CRC Press, Boca Raton, FL, 2011).

83.Ph.L. Gould: Introduction to Linear Elasticity (Springer, Heidelberg, New York, 2013). 